\title{
Dwell Time Optimization in Switching Control of Parameter Varying Time Delay Systems
}

Peng Yan

\author{
Hitay Özbay
}

\author{
Murat Şansal
}

\begin{abstract}
It has been shown that parameter varying systems with time delays can be robustly stabilized by switching control, provided that the plant's parameter varies slowly enough such that the dwell time conditions of the switched controllers can be satisfied. In this paper, the minimization of dwell time is considered, where an iterative search algorithm is developed from the singular value perspectives. The local minimal dwell time obtained in this paper can be used to estimate the upper bound on how fast the plant's parameters can vary. Meanwhile, the switching controller synthesis with optimal dwell time is also discussed, where robust stabilizer design algorithm is presented to achieve robust stability at certain operating range, as well as the local minimal dwell time for controller switching. A numerical example is given to illustrate the proposed algorithm.
\end{abstract}

\section{INTRODUCTION}

The control of time delay systems has many important applications in various engineering fields such as chemical processes, aerodynamics, and communication networks [4], [15]. Due to infinite dimensionality of the state space, delay systems pose challenging control problems [8], [7], [9], [15]. Furthermore, many time delay systems are time varying and parameter dependent, where system parameters are scheduled along a measurable parameter trajectory [20], [24], [28]. An example of parameter varying time delay systems is the data congestion control model for TCP networks, where all the parameters of the dynamical model, including the time delay $R T T$ (round trip time), are dependent on instantaneous queue length at the bottleneck network node [14], [29].

The analysis and control of LPV (Linear Parameter Varying) delay free systems have been discussed widely [1], [20], [24], among which an important method is switching control, which employs multiple candidate controllers at different operating ranges and use switching logic to select the active controller at each instant of time. We refer to [5], [6], [11], [12], [19], [30] and references therein for hybrid system stability analysis and switching control synthesis for systems without time delays. It is worth noting that the approach of switching control is extended to LPV systems with unknown time-varying parameters in a recent result [27], where the model being considered is linear time-varying without time delays.

P. Yan is with Systems Department, United Technologies Research Center, 411 Silver Lane, East Hartford, CT, 06108, USA yanpeutrc.utc.com; also with School of Mechanical Engineering, Shandong University, Jinan, Shandong, 250061, P.R.China

H. Özbay is with Dept. of Electrical \& Electronics Engineering, Bilkent University, Bilkent, Ankara 06800, Turkey, hitay@bilkent.edu.tr

M. Şansal is with Meteksan Defence Industry Inc., Beytepe Köyü Yolu No:3, Bilkent, 06800 Ankara, Turkey msansal@meteksansavunma.com.tr
There are also various recent results on LPV time delay systems [17], [21], [28]. In [21] stability and stabilization were discussed for discrete time switched time delay systems; [17] considered similar stability problem in continuous time domain. Note that [21] and [17] are trajectory dependent results without taking admissible switching signals into considerations. Meanwhile, trajectory independent switching was discussed in [32], which showed that robust stabilization can be achieved using switching control for LPV time delay systems if the plant's parameter varies slow enough to meet the dwell time conditions. An open problem is the minimization of the dwell time in the synthesis of switching controllers, such that faster switching can be allowed in control applications.

We present in this paper a numerical algorithm to minimize the dwell time between switching instants, and to design the corresponding controllers. Compared to the switching dwell time for delay free systems [10], [19], the dwell time conditions derived for time delay systems are more conservative [31]. Therefore the minimization of the dwell time in switching controller synthesis is of particular importance from application perspectives. The stability conditions derived in [2], [31], [32] are taken as the basis here. Hence the present work complements these papers.

The paper is organized as follows. Some preliminaries are given in Section II, where robust stabilization problem is defined for parameter varying time delay systems with switched controllers. In Section III, a numerical algorithm is presented to minimize the dwell time, with which the stabilizer synthesis is also considered to meet the minimal dwell time requirements, besides robust stability conditions. Numerical example is given in Section IV, followed by concluding remarks in Section V.

\section{PRELIMINARIES}

Consider the following linear parameter varying time delay system $\Sigma_{\theta}$ for $t \geq 0$ :

$\Sigma_{\theta}:\left\{\begin{array}{c}\dot{x}(t)=A(\theta) x(t)+\bar{A}(\theta) x(t-\tau(\theta))+B(\theta) u(t) \\ x_{0}(\xi)=\phi(\xi), \quad \forall \xi \in\left[-\tau_{\max }, 0\right]\end{array}\right.$

where $x(t) \in \mathbb{R}^{n}$ is the state vector, $u(t) \in \mathbb{R}^{m}$ is control input, $\tau(\theta)$ denotes the parameter varying time-delay satisfying $0<\tau(\theta) \leq \tau_{\max }$, and $d \tau / d t=d \tau / d \theta * d \theta / d t<1$. The LPV time delay system $\Sigma_{\theta}$ depends on a parameter $\theta(t)$, where $\theta(t) \in \mathbb{R}$ is assumed to be continuously differentiable and $\theta \in \Theta$ where $\Theta$ is a compact set.

As depicted in Fig. 1, a switching control approach was discussed in [32], where $K_{i}$ is a state feedback controller de- 
signed for operating points $\theta=\theta_{i}$, which robustly stabilizes the LPV time delay systems for

$$
\theta \in \Theta_{i}:=\left[\theta_{i}^{-}, \theta_{i}^{+}\right]
$$

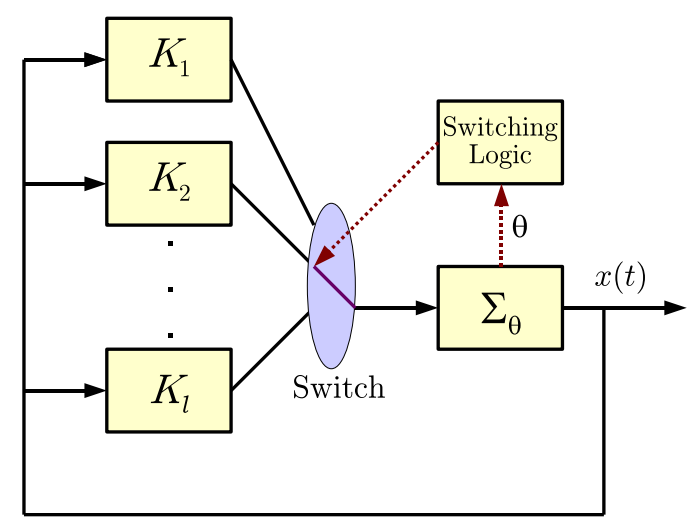

Fig. 1. The switched feedback control system

The feedback system equation can be written as:

$$
\Sigma_{q}:\left\{\begin{array}{c}
\dot{x}(t)=A_{q(t)}^{c}(\theta) x(t)+\bar{A}(\theta) x(t-\tau(\theta)), t \geq 0 \\
x_{0}(\xi)=\phi(\xi), \quad \forall \xi \in\left[-\tau_{\max }, 0\right]
\end{array}\right.
$$

where $A_{q(t)}^{c}(\theta)=A(\theta)+B(\theta) K_{q(t)}$ and $q(t)$ is a piecewise switching signal taking values on the set $\mathcal{F}:=\{1,2, \ldots, l\}$, i.e. $q(t)=k_{j}, k_{j} \in \mathcal{F}$, for $\forall t \in\left[t_{j}, t_{j+1}\right)$, where $t_{j}, j \in$ $\mathbb{Z}^{+} \cup\{0\}$, is the $j^{\text {th }}$ switching time instant which applies controller $K_{k_{j}}, u=K_{k_{j}} x$ for $\theta \in \Theta_{k_{j}}$.

The notation used in this paper is the same as [32]: $\|\cdot\|$ denotes the Euclidean norm in $\mathbb{R}^{n}$, and for a continuous function $f \in C\left([t-r, t], \mathbb{R}^{n}\right)$ we define

$$
|f|_{[t-r, t]}:=\sup _{t-r \leq \theta \leq t}\|f(\theta)\| .
$$

The switched time-delay system $\Sigma_{q}$ described by (3) is stable if there exists a continuous strictly increasing function $\bar{\alpha}: \mathbb{R}^{+} \rightarrow \mathbb{R}^{+}$with $\bar{\alpha}(0)=0$ such that

$$
\|x(t)\| \leq \bar{\alpha}\left(|x|_{\left[t_{0}-\tau_{\max }, t_{0}\right]}\right), \quad \forall t \geq t_{0} \geq 0,
$$

along the trajectory of (3). Furthermore, $\Sigma_{q}$ is asymptotically stable when $\Sigma_{q}$ is stable and $\lim _{t \rightarrow+\infty} x(t)=0$.

For a given positive constant $\tau_{D}$, the switching signal set based on the dwell time $\tau_{D}$ is denoted by $S\left[\tau_{D}\right]$, where for any switching signal $q(t) \in S\left[\tau_{D}\right]$, the distance between any consecutive discontinuities of $q(t), t_{j+1}-t_{j}, j \in \mathbb{Z}^{+} \cup\{0\}$, is larger than $\tau_{D}$ [10], [22].

Recall the main results of [32]. Consider the trajectory of (3) in an arbitrary switching interval $t \in\left[t_{j}, t_{j+1}\right)$ :

$$
\Sigma_{k_{j}}:\left\{\begin{aligned}
\dot{x}(t)= & \left(A_{k_{j}}^{c}+\Delta A_{k_{j}}^{c}(\theta)\right) x(t) \\
& +\left(\bar{A}_{k_{j}}+\Delta \bar{A}(\theta)\right) x\left(t-\tau_{k_{j}}(\theta)\right) \\
x_{t_{j}}(\xi)= & \phi_{j}(\xi), \quad \forall \xi \in\left[-\bar{\tau}_{k_{j}}, 0\right],
\end{aligned}\right.
$$

where $0<\bar{\tau}_{k_{j}}:=\max \tau_{k_{j}}(\theta)$, for $\theta \in \Theta_{k_{j}}, \phi_{j}(\xi)$ is defined as:

$$
\phi_{j}(\xi)=\left\{\begin{array}{lr}
x\left(t_{j}+\xi\right) & -\bar{\tau}_{k_{j}} \leq \xi<0 \\
\lim _{h \rightarrow 0^{-}} x\left(t_{j}+h\right), & \xi=0
\end{array}\right.
$$

Construct the Lyapunov-Razumikhin function

$$
V_{k_{j}}\left(x_{j}, t\right)=x_{j}^{T}(t) P_{k_{j}} x_{j}(t), \quad t \in\left[t_{j}, t_{j+1}\right]
$$

for (5), then we have

$$
\kappa_{k_{j}}\left\|x_{j}(t)\right\|^{2} \leq V_{k_{j}}\left(t, x_{j}\right) \leq \bar{\kappa}_{k_{j}}\left\|x_{j}(t)\right\|^{2}, \forall x_{j} \in \mathbb{R}^{n},
$$

where $\kappa_{k_{j}}:=\sigma_{\min }\left[P_{k_{j}}\right]>0$ denotes the smallest singular value of $P_{k_{j}}$ and $\bar{\kappa}_{k_{j}}:=\sigma_{\max }\left[P_{k_{j}}\right]>0$ the largest singular value of $P_{k_{j}}$.

As in [32], assuming existence of a constant $p_{k_{j}}>1$ satisfying $V_{k_{j}}\left(t+\varphi, x_{j}(t+\varphi)\right)<p_{k_{j}} V_{k_{j}}\left(t, x_{j}(t)\right)$ for $\forall \varphi \in$ $\left[-2 \bar{\tau}_{k_{j}}, 0\right]$, we obtain

$$
\dot{V}_{k_{j}}\left(t, x_{j}\right) \leq-x_{j}^{T}(t) S_{k_{j}} x_{j}(t),
$$

with

$$
\begin{aligned}
S_{k_{j}}:= & -\left\{S^{1}+S^{2}+S^{3}+\gamma_{k_{j}} P_{k_{j}} D_{k_{j}} D_{k_{j}}^{T} P_{k_{j}}\right. \\
& +\bar{\gamma}_{k_{j}} P_{k_{j}} \bar{D}_{k_{j}} \bar{D}_{k_{j}}^{T} P_{k_{j}}+\bar{\gamma}_{k_{j}}^{-1} \bar{E}_{k_{j}}^{T} \bar{E}_{k_{j}}+2 \bar{\tau}_{k_{j}} p_{k_{j}} P_{k_{j}} \\
& \left.+\bar{\tau}_{k_{j}} P_{k_{j}}\left(\bar{A}_{k_{j}}\left(Q_{k_{j}}+\bar{Q}_{k_{j}}\right) \bar{A}_{k_{j}}^{T}+\epsilon_{k_{j}} \bar{D}_{k_{j}} \bar{D}_{k_{j}}^{T}\right) P_{k_{j}}\right\}
\end{aligned}
$$

where

$$
\begin{aligned}
S^{1}= & P_{k_{j}}\left(A_{k_{j}}+B_{k_{j}} K_{k_{j}}+\bar{A}_{k_{j}}\right) \\
& +\left(A_{k_{j}}+B_{k_{j}} K_{k_{j}}+\bar{A}_{k_{j}}\right)^{T} P_{k_{j}} \\
S^{2}= & \gamma_{k_{j}}^{-1}\left(E_{k_{j}}+E_{k_{j}}^{B} K_{k_{j}}\right)^{T}\left(E_{k_{j}}+E_{k_{j}}^{B} K_{k_{j}}\right) \\
S^{3}= & \bar{\tau}_{k_{j}} P_{k_{j}} \bar{A}_{k_{j}}\left(Q_{k_{j}}+\bar{Q}_{k_{j}}\right) \bar{E}_{k_{j}}^{T}\left(\epsilon_{k_{j}} I-\bar{E}_{k_{j}}\left(Q_{k_{j}}\right.\right. \\
& \left.\left.+\bar{Q}_{k_{j}}\right) \bar{E}_{k_{j}}^{T}\right)^{-1} \bar{E}_{k_{j}}\left(Q_{k_{j}}+\bar{Q}_{k_{j}}\right) \bar{A}_{k_{j}}^{T} P_{k_{j}}
\end{aligned}
$$

and $\gamma_{k_{j}}>0, \bar{\gamma}_{k_{j}}>0, \epsilon_{k_{j}}>0$ are free scalars, and $Q_{k_{j}}>$ $0, \bar{Q}_{k_{j}}>0$ are chosen such that

$$
\begin{aligned}
\left(( A _ { k _ { j } } ^ { c } + \Delta A _ { k _ { j } } ^ { c } ( \theta + \varphi ) ) ^ { T } Q _ { k _ { j } } ^ { - 1 } \left(\left(A_{k_{j}}^{c}+\Delta A_{k_{j}}^{c}(\theta+\varphi)\right)\right.\right. & \leq P_{k_{j}} \\
\left(\bar{A}_{k_{j}}+\Delta \bar{A}(\theta+\varphi)\right)^{T} \bar{Q}_{k_{j}}^{-1}\left(\bar{A}_{k_{j}}+\Delta \bar{A}(\theta+\varphi)\right) & \leq P_{k_{j}} .
\end{aligned}
$$

The following result can be obtained by defining $X_{k_{j}}=$ $P_{k_{j}}^{-1}$ and employing Schur complement and Razumikhin theorem [2], [32]:

Lemma 2.1: The time varying time delay system (5) is robustly stable if there exist $X_{k_{j}}>0, Q_{k_{j}}>0, \bar{Q}_{k_{j}}>0$, $Y_{k_{j}}$, and scalars $\gamma_{k_{j}}>0, \bar{\gamma}_{k_{j}}>0, \epsilon_{k_{j}}>0, \rho_{k_{j}}>0$, $\bar{\rho}_{k_{j}}>0$, such that

$$
\left[\begin{array}{ccc}
X_{k_{j}} & X_{k_{j}} A_{k_{j}}^{T}+Y_{k_{j}}^{T} B_{k_{j}}^{T} & X_{k_{j}} E_{k_{j}}^{T}+Y_{k_{j}}^{T}\left(E_{k_{j}}^{B}\right)^{T} \\
\star & Q_{k_{j}}-\rho_{k_{j}} D_{k_{j}} D_{k_{j}}^{T} & 0 \\
\star & \star & \rho_{k_{j}} I
\end{array}\right] \geq 0
$$

$$
\begin{gathered}
{\left[\begin{array}{cccc}
X_{k_{j}} & X_{k_{j}} \bar{A}_{k_{j}}^{T} & X_{k_{j}} \bar{E}_{k_{j}}^{T} \\
\star & \bar{Q}_{k_{j}}-\bar{\rho}_{k_{j}} \bar{D}_{k_{j}} \bar{D}_{k_{j}}^{T} & 0 \\
\star & \star \star & \bar{\rho}_{k_{j}} I
\end{array}\right] \geq 0} \\
{\left[\begin{array}{cccc}
M_{k_{j}} & R_{12} & X_{k_{j}} \bar{E}_{k_{j}}^{T} & R_{14} \\
\star & -\gamma_{k_{j}} I & 0 & 0 \\
\star & \star & -\bar{\gamma}_{k_{j}} I & 0 \\
\star & \star & \star & N_{k_{j}}
\end{array}\right]<0}
\end{gathered}
$$


where

$$
\begin{gathered}
R_{12}:=X_{k_{j}} E_{k_{j}}^{T}+Y_{k_{j}}^{T}\left(E_{k_{j}}^{B}\right)^{T} \\
R_{14}:=\bar{\tau}_{k_{j}} \bar{A}_{k_{j}}\left(Q_{k_{j}}+\bar{Q}_{k_{j}}\right) \bar{E}_{k_{j}}^{T} \\
M_{k_{j}}=\left(A_{k_{j}}+\bar{A}_{k_{j}}\right) X_{k_{j}}+X_{k_{j}}\left(A_{k_{j}}+\bar{A}_{k_{j}}\right)^{T} \\
+\gamma_{k_{j}} D_{k_{j}} D_{k_{j}}^{T}+\bar{\gamma}_{k_{j}} \bar{D}_{k_{j}} \bar{D}_{k_{j}}^{T}+B_{k_{j}} Y_{k_{j}}+Y_{k_{j}}^{T} B_{k_{j}}^{T} \\
+\bar{\tau}_{k_{j}} \epsilon_{k_{j}} \bar{D}_{k_{j}} \bar{D}_{k_{j}}^{T}+\bar{\tau}_{k_{j}} \bar{A}_{k_{j}}\left(Q_{k_{j}}+\bar{Q}_{k_{j}}\right) \bar{A}_{k_{j}}^{T}+2 \bar{\tau}_{k_{j}} p_{k_{j}} X_{k_{j}}, \\
N_{k_{j}}=-\bar{\tau}_{k_{j}}\left(\epsilon_{k_{j}} I-\bar{E}_{k_{j}}\left(Q_{k_{j}}+\bar{Q}_{k_{j}}\right) \bar{E}_{k_{j}}^{T}\right),
\end{gathered}
$$

and $\star$ denotes the transpose of the symmetric term in symmetric matrices. We refer to [32] for the definitions of $D_{k_{j}}, \bar{D}_{k_{j}}, E_{k_{j}}, \bar{E}_{k_{j}}, E_{k_{j}}^{B}$. Furthermore, the state feedback controller

$$
K_{k_{j}}=Y_{k_{j}} X_{k_{j}}^{-1}
$$

is robustly stabilizing $\Sigma_{k_{j}}$, (5).

The stability of the switched LPV time delay system (3) can be guaranteed with the following dwell time condition [32]:

Lemma 2.2: Consider switched LPV time delay system (3) with $l$ state feedback controllers designed for $\theta \in \Theta_{i}$, $i \in \mathcal{F}$ as described by (2), where each controller $K_{k_{j}}, k_{j} \in$ $\mathcal{F}$, is a robustly stabilizing controller derived from Lemma 2.1. Let the dwell time be defined by

$$
\tau_{D}:=T^{*}+2 \tau_{\max }
$$

where

$$
T^{*}:=\lambda \mu\left\lfloor\frac{\lambda-1}{\bar{p}-1}+1\right\rfloor,
$$

with $\bar{p}:=\min _{k_{j} \in \mathcal{F}}\left\{p_{k_{j}}\right\}>1,\lfloor\cdot\rfloor$ being the floor integer function, and

$$
\lambda:=\max _{k_{j} \in \mathcal{F}} \frac{\bar{\kappa}_{k_{j}}}{\kappa_{k_{j}}},
$$

and

$$
\mu:=\max _{k_{j} \in \mathcal{F}} \frac{\bar{\kappa}_{k_{j}}}{w_{k_{j}}}
$$

where

$$
w_{k_{j}}:=\sigma_{\min }\left[S_{k_{j}}\right]>0
$$

Then system (3) is asymptotically stable for any switching rule $q(t) \in S\left[\tau_{D}\right]$.

\section{Dwell Time Minimization}

Due to the free parameters of $p_{i}, X_{i}, Q_{i}, \bar{Q}_{i}, Y_{i}, \gamma_{i}, \bar{\gamma}_{i}, \epsilon_{i}$, $\rho_{i}, \bar{\rho}_{i}$ in Lemma 2.1, the candidate stabilizer design is not unique. As illustrated by the numerical example discussed in [32], the dwell time computation depends heavily on the selection of the free parameters. It is a very challenging question to optimize the design of the stabilizers such that the minimal dwell time can be achieved to tolerate faster parameter variations of LPV time delays systems $\Sigma_{\theta}$. In this section, we will provide a search algorithm for numerical optimization of the dwell time, (14)

Consider an arbitrary switching time instant $j^{t h}, j \in \mathbb{Z}^{+} \cup$ $\{0\}$, where controller $K_{k_{j}}$ is applied for $\forall t \in\left[t_{j}, t_{j+1}\right)$.
Define $\Pi_{k_{j}}^{p_{k_{j}}}$ for the LMIs of (11-13) in Lemma 2.1. Using the same arguments in Lemma 2.1, we denote

$$
\Xi_{k_{j}}^{(z, p)}=\left[\begin{array}{ccccc}
M_{k_{j}}^{p} & R_{12} & X_{k_{j}} \bar{E}_{k_{j}}^{T} & R_{14} & X_{k_{j}} \\
\star & -\gamma_{k_{j}} I & 0 & 0 & 0 \\
\star & \star & -\bar{\gamma}_{k_{j}} I & 0 & 0 \\
\star & \star & \star & N_{k_{j}} & 0 \\
\star & \star & \star & \star & -z I
\end{array}\right]
$$

where

$$
\begin{aligned}
M_{k_{j}}^{p} & =\left(A_{k_{j}}+\bar{A}_{k_{j}}\right) X_{k_{j}}+X_{k_{j}}\left(A_{k_{j}}+\bar{A}_{k_{j}}\right)^{T} \\
& +\gamma_{k_{j}} D_{k_{j}} D_{k_{j}}^{T}+\bar{\gamma}_{k_{j}} \bar{D}_{k_{j}} \bar{D}_{k_{j}}^{T}+B_{k_{j}} Y_{k_{j}}+Y_{k_{j}}^{T} B_{k_{j}}^{T} \\
& +\bar{\tau}_{k_{j}} \epsilon_{k_{j}} \bar{D}_{k_{j}} \bar{D}_{k_{j}}^{T}+\bar{\tau}_{k_{j}} \bar{A}_{k_{j}}\left(Q_{k_{j}}+\bar{Q}_{k_{j}}\right) \bar{A}_{k_{j}}^{T}+2 \bar{\tau}_{k_{j}} p X_{k_{j}} .
\end{aligned}
$$

For $\forall z>0$, we claim that $\Xi_{k_{j}}^{\left(z, p_{k_{j}}\right)}<0$ is necessary and sufficient to guarantee $S_{k_{j}}>z^{-1} I$. Note that

$$
\begin{aligned}
& S_{k_{j}}>z^{-1} I \\
\Longleftrightarrow & S_{k_{j}}-z^{-1} I>0 \\
\Longleftrightarrow & X_{k_{j}}\left(S_{k_{j}}-z^{-1} I\right) X_{k_{j}}>0 \\
\Longleftrightarrow & X_{k_{j}}\left(-S_{k_{j}}\right) X_{k_{j}}+X_{k_{j}} z^{-1} X_{k_{j}}<0
\end{aligned}
$$

Recall (10), we can derive the following inequalities from Schur complements:

$$
X_{k_{j}}\left(-S_{k_{j}}\right) X_{k_{j}}+X_{k_{j}} z^{-1} X_{k_{j}}<0 \Longleftrightarrow \Xi_{k_{j}}^{\left(z, p_{k_{j}}\right)}<0 .
$$

Now we are ready to state the numerical search algorithm to minimize the dwell time $\tau_{D}$ given in (14). In the following search algorithm, we assume the existence of the dwell time, i.e. feasible solutions of LMIs of $\Pi_{k_{j}}^{p_{k_{j}}}$.

Step 1. Initialize the search with $p^{*}=p_{0}>1$, and $z^{*}=$ $z_{0}>0$, where a sufficiently small $p_{0}>1$ and a sufficiently large $z_{0}>0$ can be chosen as a feasible initial condition.

Step 2.Let $p_{k_{j}}=p^{*}$, solve the following LMIs:

$$
\begin{aligned}
0 & <z \\
z & <z^{*} \\
\Xi_{k_{j}}^{\left(z, p^{*}\right)} & <0 \\
I & <X_{k_{j}}
\end{aligned}
$$

and

$$
\Pi_{k_{j}}^{p^{*}} \text { are satisfied, } \forall k_{j} \in \mathcal{F}
$$

It is worth noting that the LMI condition of $X_{k_{j}}>I$ normalizes the search. If feasible solutions exist, go to step 3 , otherwise skip to step 4.

Step 3. Let $p^{*}=p^{*}+\delta p$ and $z^{*}=z$, go to step 1 to find a smaller $z$, where $\delta p$ is the step size to search $p^{*}$ incrementally.

Step 4. Iterate step 2 and 3 until the LMI solver could not find feasible solutions. We denote $\mathbf{z}^{*}=\min z^{*}$ and $\mathbf{p}^{*}=$ $\max p^{*}$.

Step 5. For $\forall k_{j} \in \mathcal{F}$, deploy the following optimization subject to LMI conditions: 


$$
\begin{array}{cc}
\min & \nu_{k_{j}}^{+} \\
\text {s.t. } & 0<\nu_{k_{j}}^{+} \text {is a scalar } \\
& \Xi_{k_{j}}^{\left(\mathbf{z}^{*}, \mathbf{p}^{*}\right)}<0 \\
I<X_{k_{j}} \\
X_{k_{j}}<\nu_{k_{j}}^{+} I \\
\Pi_{k_{j}}^{\mathbf{p}^{*}} \text { are satisfied }
\end{array}
$$

Denote $\bar{\nu}_{k_{j}}^{+}=\min \left\{\nu_{k_{j}}^{+}\right\}$, and solve the following LMI optimization problem:

$$
\begin{array}{cc}
\max & \nu_{k_{j}}^{-} \\
\text {s.t. } & 0<\nu_{k_{j}}^{-} \text {is a scalar } \\
& \Xi_{k_{j}}^{\left(\mathbf{z}^{*}, \mathbf{p}^{*}\right)}<0 \\
\nu_{k_{j}}^{-} I<X_{k_{j}} \\
X_{k_{j}}<\bar{\nu}_{k_{j}}^{+} I \\
\Pi_{k_{j}}^{\mathbf{p}^{*}} \text { are satisfied }
\end{array}
$$

We denote $\bar{\nu}_{k_{j}}^{-}=\max \left\{\nu_{k_{j}}^{-}\right\}$, and $p^{*}, X_{k_{j}}^{*}, Q_{k_{j}}^{*}, \bar{Q}_{k_{j}}^{*}, Y_{k_{j}}^{*}$, $\gamma_{k_{j}}^{*}, \bar{\gamma}_{k_{j}}^{*}, \epsilon_{k_{j}}^{*}, \rho_{k_{j}}^{*}, \bar{\rho}_{k_{j}}^{*}$ the corresponding solution of LMI set (22). An upper bound of the minimal dwell time can be derived:

Theorem 3.1: Follow the above search algorithm step 1 to 5 ; the resulting minimal dwell time $\tau_{D}$ is bounded by:

$$
\tau_{D}<\tau_{D}^{*}:=\frac{\lambda^{*} \mathbf{z}^{*}}{\eta^{*}}\left\lfloor\frac{\lambda^{*}-1}{\mathbf{p}^{*}-1}+1\right\rfloor+2 \tau_{\max },
$$

where

$$
\lambda^{*}:=\max _{k_{j} \in \mathcal{F}} \frac{\bar{\nu}_{k_{j}}^{+}}{\bar{\nu}_{k_{j}}^{-}}
$$

and

$$
\eta^{*}:=\min _{k_{j} \in \mathcal{F}} \bar{\nu}_{k_{j}}^{-}
$$

Meanwhile corresponding robust stabilizers are derived from:

$$
K_{k_{j}}^{*}=Y_{k_{j}}^{*}\left(X_{k_{j}}^{*}\right)^{-1}
$$

Proof. Recall that $P_{k_{j}}=X_{k_{j}}^{-1}$, and observe $\bar{\nu}_{k_{j}}^{-}<X_{k_{j}}<$ $\bar{\nu}_{k_{j}}^{+}$. We have:

$$
\left(\bar{\nu}_{k_{j}}^{+}\right)^{-1}<P_{k_{j}}<\left(\bar{\nu}_{k_{j}}^{-}\right)^{-1}
$$

The singular value ratio $\lambda$ in (16) can be bounded by:

$$
\lambda<\lambda^{*}:=\max _{k_{j} \in \mathcal{F}} \frac{\bar{\nu}_{k_{j}}^{+}}{\bar{\nu}_{k_{j}}^{-}} .
$$

From the definition of $\mu$ in (17) and the fact that $\left(\mathbf{z}^{*}\right)^{-1}<$ $\sigma_{\min }\left[S_{k_{j}}\right]$, we have:

$$
\begin{aligned}
\mu & <\mathbf{z}^{*} \max _{k_{j} \in \mathcal{F}} \bar{\kappa}_{k_{j}} \\
& <\mathbf{z}^{*} \max _{k_{j} \in \mathcal{F}}\left(\bar{\nu}_{k_{j}}^{-}\right)^{-1} \\
& =\mathbf{z}^{*} \frac{1}{\min _{k_{j} \in \mathcal{F}} \bar{\nu}_{k_{j}}^{-}} \\
& =\frac{\mathbf{z}^{*}}{\eta^{*}}
\end{aligned}
$$

which implies (23) and completes the proof.

Note that the search of an optimal free parameter $p^{*}$ can not be deployed from LMI optimization perspectives due to the term of $2 \bar{\tau}_{k_{j}} p^{*} X_{k_{j}}$ in $\Xi_{k_{j}}^{(z, p)}$. Meanwhile, it is worth mentioning that the conservatism of the above search algorithm is mainly due to the assumption of $p_{k_{j}}=p^{*}$ for the convenience of computation. It is possible to generate better results with optimization over multi-dimensional space of $\left(p_{1}, p_{2}, \cdots, p_{l}\right)$. The above algorithm can be deployed using Matlab ${ }^{\circledR}$ and its Robust Control ToolBox.

\section{NUMERICAL EXAMPLE}

In this part of the paper, the example of [32] is considered to illustrate the algorithm discussed in Section III. The parameters of (1) be given as

$$
\begin{gathered}
A(\theta)=\left[\begin{array}{rr}
-2.5-1 \theta & -0.75-0.5 \theta \\
-1 & -1.95+0.1 \theta
\end{array}\right] \\
\bar{A}(\theta)=\left[\begin{array}{rr}
-1 & 0 \\
-0.2-0.5 \theta & -1
\end{array}\right] \quad B(\theta)=\left[\begin{array}{ll}
1 & 1
\end{array}\right]^{T}
\end{gathered}
$$

$\tau(\theta)=0.15-0.05 \theta$ and $\theta(t)=\cos \left(\omega_{o} t\right)$, Note that $\theta \in$ $[-1,1]=\Theta$, and $\omega_{o}$ determines the speed of parameter variations.

Similar to [32], we define $\theta_{1}=0.5, \theta_{2}=-0.5$ and two parameter intervals

$$
\Theta_{1}=[-0.1,1] \quad \Theta_{2}=[-1,0.1]
$$

for which two separate controllers $K_{1}$ and $K_{2}$ are to be designed and switched according to hysteresis switching logic with the hysteresis range $\Theta_{1} \cap \Theta_{2}$ defined as $\left|d_{1,2}\right|=$ 0.2 . The two nominal systems are defined as:

$$
\begin{gathered}
A_{1}=\left[\begin{array}{rr}
-3 & -1 \\
-1 & -1.9
\end{array}\right] \quad \bar{A}_{1}=\left[\begin{array}{rr}
-1 & 0 \\
-0.45 & -1
\end{array}\right] \\
A_{2}=\left[\begin{array}{rr}
-2 & -0.5 \\
-1 & -2
\end{array}\right] \quad \bar{A}_{2}=\left[\begin{array}{rr}
-1 & 0 \\
0.05 & -1
\end{array}\right] \\
E_{1}=E_{2}=\left[\begin{array}{rr}
0.6 & 0.3 \\
0 & 0.06
\end{array}\right] \quad \bar{E}_{1}=\bar{E}_{2}=\left[\begin{array}{rr}
0 & 0 \\
0.3 & 0
\end{array}\right]
\end{gathered}
$$

$D_{1}=D_{2}=\bar{D}_{1}=\bar{D}_{2}=I$, with $\bar{\tau}_{1}=\max _{\theta \in \Theta_{1}}=0.155$ sec., $\bar{\tau}_{2}=\max _{\theta \in \Theta_{2}}=0.20 \mathrm{sec}$.

We now pick up arbitrary initial conditions $p_{0}=2.4$ and $z_{0}=4$ in step 1 and perform the iterative search of step 2 and 3. We have $\mathbf{z}^{*}=3.145$ and $\mathbf{p}^{*}=2.700$.

By further deploying the LMI optimization (21) and (22) in step 5 with the achieved $\mathbf{z}^{*}$, and $\mathbf{p}^{*}$, we can synthesize the two stabilizers:

$$
K_{1}=\left[\begin{array}{ll}
0.9681 & 0.0465
\end{array}\right] \quad K_{2}=\left[\begin{array}{ll}
-0.2708 & 0.3715
\end{array}\right]
$$

and

$$
\begin{aligned}
& \lambda=\max \left\{\bar{\kappa}_{1} / \kappa_{1}, \bar{\kappa}_{2} / \kappa_{2},\right\}=1.3807 \\
& \mu=\max \left\{\bar{\kappa}_{1} / \omega_{1}, \bar{\kappa}_{2} / \omega_{2},\right\}=0.3772
\end{aligned}
$$


The dwell time can be calculated from (15) and (14):

$$
\begin{aligned}
\tau_{D} & =2 \tau_{\max }+\lambda \mu\left\lfloor\frac{\lambda-1}{\bar{p}-1}+1\right\rfloor \\
& =2 * 0.2+1.3807 * 0.3772\left\lfloor\frac{1.3807-1}{2.700-1}+1\right\rfloor \\
& =0.92
\end{aligned}
$$

Meanwhile, we have:

$$
\begin{gathered}
\lambda^{*}=\max \left\{\bar{\nu}_{1}^{+} / \bar{\nu}_{1}^{-}, \bar{\nu}_{2}^{+} / \bar{\nu}_{2}^{-}\right\}=1.3858 \\
\eta^{*}=\min \left\{\bar{\nu}_{1}^{-}, \bar{\nu}_{2}^{-}\right\}=0.9996
\end{gathered}
$$

so, from Theorem 3.1, an upper bound for the minimal dwell time can be given as:

$$
\tau_{D}^{*}=\frac{\lambda^{*} \mathbf{z}^{*}}{\eta^{*}}\left\lfloor\frac{\lambda^{*}-1}{\mathbf{p}^{*}-1}+1\right\rfloor+2 \tau_{\max }=4.76
$$

The level of conservatism in Theorem 3.1 is illustrated by the amount of deviation of $\tau_{D}^{*}$ from $\tau_{D}$.

For comparison purpose, we also use the above parameters in the approach of the earlier paper [32], which generates the controllers

$$
K_{1}=\left[\begin{array}{ll}
1.2651 & 0.8975
\end{array}\right] \quad K_{2}=\left[\begin{array}{ll}
0.2122 & 0.4232
\end{array}\right]
$$

and gives the corresponding dwell time as

$$
\begin{aligned}
\tilde{\tau}_{D} & =2 \tau_{\max }+\lambda \mu\left\lfloor\frac{\lambda-1}{\bar{p}-1}+1\right\rfloor \\
& =2 * 0.2+1.7308 * 0.5843\left\lfloor\frac{1.7308-1}{2.700-1}+1\right\rfloor \\
& =1.41
\end{aligned}
$$

In view of $0.92<1.41$, clearly the algorithm proposed in the present paper can derive a smaller dwell time. Recall Corollary 3.3 in [32], we can guarantee switching stability with

$$
|\dot{\theta}|<0.2 / 0.92 \approx 0.2174
$$

which implies that $\omega_{o} \in(0,0.2174)$. However, the dwell time $\tilde{\tau}_{D}$ derived from [32] only allows much slower parameter variations:

$$
|\dot{\theta}|<0.2 / 1.41 \approx 0.1418
$$

i.e. $\omega_{0} \in(0,0.1418)$.

We should also indicate that the above results can be further improved by a selection of a suitable initial values $p_{0}$ and $z_{0}$ as shown in Table I, which includes a summary of the above results.

\section{CONCLUSIONS}

This paper considered the minimization of dwell time in robust stabilization of time varying time delay systems with switched controllers. Compared to the earlier results [31] and [32], the algorithm obtained in the present paper allows for faster parameter variation and therefore faster switchings. An iterative search algorithm is proposed based on the optimization of matrix singular values using LMIs. This procedure is guaranteed to minimize an upper bound of the dwell time, $\tau_{D}^{*}$, but the actual dwell time $\tau_{D}$ obtained by the switching robust controllers designed using the parameters of this optimization can be much lower as illustrated by the numerical example.

In conclusion, this paper provides a methodology for the design of switching robust controllers with the objective of making the dwell time as small as possible. Although there is still room for improvement in the controller design for the minimal achievable dwell time, the present paper provides a basis on which new methods can be built and compared with.

\section{REFERENCES}

[1] P. Apkarian, P. Gahinet and G. Becker, "Self-scheduled $\mathcal{H}^{\infty}$ control of linear parameter-varying systems: a design example", Automatica, 31:1251-1261, 1995.

[2] Y.-Y. Cao, Y.-X. Sun, and C. Cheng, "Delay-dependent robust stabilization of uncertain systems with multiple state delays", IEEE Trans. Automatic Control, 43:1608-1612, 1998.

[3] C. De Persis, R. De Santis, and S. Morse, "Supervisory control with state-dependent dwell-time logic and constraints," Automatica, 40:269-275, 2004.

[4] L. Dugard and E.I. Verriest, Eds., Stability and Control of Time-Delay Systems, Springer, London, New York, 1998.

[5] J. C. Geromel and P. Colaneri, "Stability and stabilization of continouous-time switched linear systems," SIAM J. Control and Optimization, 45:1915-1930, 2006.

[6] J. C. Geromel, P. Colaneri and P. Bolzern, "Dynamic output feedback control of switched linear systems," IEEE Trans. Automatic Control, 53:720-733, 2008.

[7] K. Gu and S.-I. Niculescu, "Survey on recent results in the stability and control of time-delay systems," ASME Journal of Dynamic Systems, Measurement, and Control, 125:158-165, 2003.

[8] K. Gu, V.L. Kharitonov, and J. Chen, Stability and Robust Stability of Time-Delay Systems, Birkhauser, Boston, 2003.

\begin{tabular}{|c|c|c|c|c|c|c|c|c|c|c|c|c|}
\hline$\left(p_{0}, z_{0}\right)$ & $\left(\mathbf{p}^{*}, \mathbf{z}^{*}\right)$ & \multicolumn{2}{|c|}{$K_{1}$} & \multicolumn{2}{|c|}{$K_{2}$} & $\tau_{D}$ & $\tau_{D}^{*}$ & $\overline{K_{1}}$ & {$[32]$} & $K_{2}$ & {$[32]$} & $\tau_{D}$ of [32] \\
\hline$(3.0,5.0)$ & $(3.0,5.0)$ & {$[1.01$} & $0.09]$ & -0.27 & $0.37]$ & 0.86 & 6.84 & {$[1.18$} & 0.80 & {$[0.08$} & 0.38 & 0.85 \\
\hline$(2.4,4.0)$ & $(2.70,3.15)$ & {$[0.97$} & $0.05]$ & -0.27 & $0.37]$ & 0.92 & 4.76 & 1.27 & 0.90 & 0.21 & 0.42 & 1.41 \\
\hline$(1.1,4.0)$ & $(1.80,1.51)$ & {$[0.97$} & $0.06]$ & -0.31 & $0.46]$ & 0.80 & 2.23 & {$[1.38$} & 0.95 & 0.56 & 0.58 & 1.18 \\
\hline$(1.1,2.5)$ & $(1.65,1.39)$ & 0.97 & $0.06]$ & -0.30 & 0.49 & 0.77 & 1.99 & 1.40 & 0.95 & 0.59 & 0.59 & 1.63 \\
\hline$(1.1,1.1)$ & $(1.15,1.10)$ & {$[0.97$} & $0.07]$ & {$[-0.31$} & $0.53]$ & 0.73 & 1.58 & {$[1.46$} & 0.98 & {$[0.67$} & 0.64 & 2.08 \\
\hline$(1.05,1.05)$ & $(1.05,1.05)$ & {$[0.96$} & $0.07]$ & -0.31 & $0.53]$ & 1.06 & 2.66 & 1.47 & 0.98 & [0.70 & 0.66 & 5.02 \\
\hline
\end{tabular}

[9] J. Hale and S. Verduyn Lunel, Introduction to Functional Differential Equations, Springer-Verlag, New York, 1993.

[10] J. Hespanha and S. Morse, "Stability of switched systems with average dwell-time", Proc. of the 38th IEEE Conf. on Decision and Contr., Phoenix AZ, December 1999, pp. 2655-2660.

[11] J. Hespanha, "Uniform stability of switched linear systems: extension of Lasalle's invariance principle," IEEE Trans. Automatic Control, 49:470-482, 2004.

[12] J. Hespanha, D. Liberzon, and S. Morse, "Overcoming the limitations of adaptive control by means of logic-based switching," System \& Control Letters, 49:49-65, 2003.

TABLE I

Numerical RESUlts FOR VARIOUS INITIAL CONDITIONS 
[13] J. Hochcerman-Frommer, S. Kulkarni, and P. Ramadge, "Controller switching based on output prediction errors," IEEE Trans. Automatic Control, 43:596-607, 1998.

[14] F. Kelly, "Mathematical modelling of the Internet," in Mathematics Unlimited - 2001 and Beyond, B. Engquist and W. Schmid, Eds., Springer-Verlag, Berlin, 2001, pp. 685-702.

[15] V.L. Kharitonov, "Robust stability analysis of time delay systems: a survey," Annual Reviews in Control, 23:185-196, 1999.

[16] V.L. Kharitonov and D. Hinrichsen, "Exponential estimates for time delay systems," System \& Control Letters, 53(5):395-405, 2004.

[17] V. Kulkarni, M. Jun, and J. Hespanha, "Piecewise quadratic Lyapunov functions for piecewise affine time delay systems," in Proc. of the American Control Conference, Boston, MA, June-July 2004, pp. 38853889.

[18] J.-W. Lee, "On uniform stabilization of discrete-time linear parametervarying control systems", IEEE Trans. Automatic Control, 51:17141721, 2006.

[19] D. Liberzon and S. Morse, "Basic problems in stability and design of switched systems," IEEE Control Systems Magazine, 19:59-70, 1999.

[20] B. Lu and F. Wu, "Switching LPV control designs using multiple parameter-dependent Lyapunov fucntions", Automatica, 40:19731980, 2004.

[21] V. Montagner, V. Leite, S. Tarbouriech, and P. Peres, "Stability and stabilizability of discrete-time switched linear systems with state delay," in Proc. of the American Control Conference, 2005, Portland OR, June 2005, pp. 3806-3811.

[22] S. Morse, "Supervisory control of families of linear set-point controllers: part 1: exact matching," IEEE Trans. Automatic Control, 41:1413-1431, 1996.

[23] S.-I. Niculescu, Delay Effects on Stability: A Robust Control Approach, Lecture Notes in Control and Information Sciences No. 269, SpringerVerlag, Heidelberg, 2001.

[24] W. Rugh and J. Shamma, "Research on gain scheduling", Automatica, 36:1401-1425, 2000

[25] A. Savkin, E. Sakfidas and R. Evans, "Robust output feedback stabilizability via controller switching", System and Control Letters, 29:81-90, 1996.

[26] E. Skafidas, R. Evans, A. Savkin, and I. Peterson, "Stability results for switched controller systems," Automatica, 35:553-564, 1999.

[27] L. Vu and D. Liberzon, "Supervisory control of uncertain linear timevarying systems," IEEE Trans. Automatic Control, 56:27-42, 2011.

[28] F. Wu and K. Grigoriadis, "LPV systems with parameter-varying time delays: analysis and control," Automatica, 37:221-229, 2001.

[29] P. Yan and H. Özbay, "Robust controller design for AQM and $\mathcal{H}^{\infty}$ performance analysis," in Advances in Communication Control Networks, S. Tarbouriech, C. Abdallah, and J. Chiasson, Eds., Lecture Notes in Control and Information Sciences, No. 308, Springer-Verlag, New York, 2005, pp. 49-64.

[30] P. Yan and H. Özbay, "On switching $\mathcal{H}^{\infty}$ controllers for a class of linear parameter varying systems," System \& Control Letters, 56(78):504-511, 2007.

[31] P. Yan and H. Özbay, "Stability analysis of switched time delay systems," SIAM Journal on Control and Optimization, 47(2):936-949, 2008.

[32] P. Yan, H. Özbay, and M. Şansal, "A Switching control approach to stabilization of parameter varying time delay systems", in Proc. of the 48th IEEE Conf. on Decision and Contr., Shanghai, China, December 2009, pp. 7222-7226. 